\title{
El consumo de tabaco y alcohol en jóvenes de zonas urbanas marginadas de México. Un análisis de decisiones relacionadas
}

\author{
José Edmundo Urquieta, SM en Econ Salud,(I) Mauricio Hernández-Avila, PhD, (I) \\ Bernardo Hernández, PhD. (I)
}

\begin{abstract}
Urquieta JE, Hernández-Avila M, Hernández B. El consumo de tabaco y alcohol en jóvenes de zonas urbanas marginadas de México. Un análisis de decisiones relacionadas. Salud Publica Mex 2006;48 supl I:S30-S40.
\end{abstract}

\section{Resumen}

Objetivo. Identificar aquellos factores económicos y sociodemográficos que influyen en la decisión de los jóvenes de fumar o consumir bebidas alcohólicas en zonas urbanas marginadas de México; probar la hipótesis de que ambas decisiones se relacionan de manera recíproca, y demostrar la influencia de las interacciones sociales al interior del hogar en el consumo de ambas sustancias. Material y métodos. Se usó la Encuesta de Evaluación de los Hogares Urbanos 2002 (Encelurb 2002), que constituye la línea basal de una encuesta de seguimiento para la evaluación del impacto de un programa social en áreas urbanas. La Encelurb 2002 es una encuesta multitemática que captó información en 17207 hogares, así como datos sobre el consumo de tabaco y alcohol en 15181 jóvenes de 12 a 21 años de edad. El método de estimación fue un modelo probit bivariado. Se analizó la muestra completa y la submuestra de 6916 adolescentes de 12 a 15 años de edad. Resultados. La estimación conjunta de las dos propensiones de consumo confirmó que ambas decisiones se relacionan en forma estrecha. La existencia de otros jóvenes mayores que fuman o que ingieren alcohol se asoció positiva y significativamente con la probabilidad de que los adolescentes de I2 a 15 años consuman ambas sustancias.A mayor edad, la probabilidad de consumir ambas sustancias adictivas es mayor, sobre todo en los hombres. Por otro lado, los jóvenes que manifestaron vivir con ambos padres tuvieron menor probabilidad de fumar o beber alcohol que aquellos que no vivían con ninguno. Conclusiones. Los resultados prueban que las decisiones de consumir tabaco o alcohol se relacionan de manera recíproca, permiten identificar mejor los fac-
Urquieta JE, Hernández-Avila M, Hernández B. Tobacco and alcohol consumption amoung youth in marginalized urban zones in Mexico.

Analysis of related decisions.

Salud Publica Mex 2006;48 suppl I:S30-S40.

\section{Abstract}

Objectives. The purpose of this study was to: identify the economic and sociodemographic factors that influence the decisions made by youth in poor urban areas in Mexico to consume tobacco and alcohol; test the hypothesis that both decisions are mutually related and; demonstrate the influence of social interactions within the household on the consumption of both substances. Material and Methods. This cross-sectional study analyzed information from the Urban Household Evaluation Survey (Encelurb 2002, per its acronym in Spanish), which constitutes the baseline measurement for a follow-up survey to evaluate the impact of a social program in urban areas of Mexico. The Encelurb 2002 is a multi-issue survey that collected information from 17 207 households, including data on alcohol and tobacco consumption from $1518 \mathrm{I}$ youth ranging in age from 12 to 21 years old. The estimation method used was a bivariate probit model. The entire sample was analyzed as well as the subsample of youth 12 to 15 years old. Results. The joint estimation of the two propensities for consumption confirmed that both decisions are closely related. The presence of older youth who smoked or consumed alcohol was positively and significantly associated with the probability of tobacco and alcohol use among youth 12 to 15 years old. The probability of consuming both addictive substances increased with age, principally among men. On the other hand, youth who lived with both parents had lower probabilities of tobacco and alcohol consumption than those living without any parent. Conclusions. The results indicate that alcohol and tobacco consumption decisions are mutually related, which allows for better identification of family and

(I) Instituto Nacional de Salud Pública. Cuernavaca, Morelos, México.

Fecha de recibido: 22 de febrero de 2006 - Fecha de aprobado: 24 de abril de 2006 Solicitud de sobretiros: Mtro. José E. Urquieta. Instituto Nacional de Salud Pública. Av. Universidad 655, Col. Sta. María Auacatitlán, 62508 Cuernavaca, Morelos, México. Correo electrónico: jurquieta@insp.mx 
tores individuales y familiares que inciden en la propensión de los jóvenes a fumar y consumir bebidas alcohólicas, y se suman a la escasa literatura en México que apunta a la necesidad de analizar el fenómeno de las adicciones en el marco del consumo de varias sustancias .

Palabras clave: jóvenes; consumo de tabaco; consumo de alcohol; modelo biprobit; corte transversal; influencia de pares; México individual factors associated with the propensity by youth for smoking and consuming alcohol. These results contribute to the scarce amount of information in Mexico indicating the need to analyze the phenomena of addictions with regard to the consumption of various substances.

Key words: youth, tobacco consumption; alcohol consumption; bivariate probit model; cross-sectional studies; peer pressure; Mexico
$\mathrm{L}$ os esfuerzos contra el consumo de sustancias adictivas que tienen probados efectos negativos en la salud se convirtieron desde hace varios años en parte importante de las políticas de salud en la mayoría de los gobiernos, entre ellos el de México. En el Programa Nacional de Salud 2001-2006, y en los programas de acción que de él se desprenden, se reconoce de manera explícita que las adicciones al tabaco y el alcohol son las principales causas de enfermedad y muerte que pueden prevenirse, y se apunta a la necesidad de la prevención y control de su consumo, en particular en los jóvenes. Las autoridades sanitarias los consideran como la población de más alto riesgo para el consumo de sustancias adictivas, pero también la población en la cual las políticas de prevención pueden ejercer un mayor efecto de control debido al poco tiempo transcurrido en el proceso de adicción. Cabe aclarar que a lo largo del texto el término consumo de tabaco se refiere al consumo de cigarrillos, que es la presentación más común del tabaco en el país.

Se estima que en México mueren 122 personas a diario por causas que se vinculan con el tabaquismo; éste es causa probable de más de 25 enfermedades y padecimientos, y se estima que los fumadores crónicos han de perder entre 20 y 25 años de vida, con la implícita carga económica a sus familias y al sector salud. ${ }^{1}$ La evidencia muestra que en México más de $60 \%$ de los fumadores inició su consumo antes de los 16 años de edad, y que más de $90 \%$ lo hizo antes de los 20 años, lo que lleva a suponer que la ventana preventiva más importante se ubica en edades tempranas, ya que rara vez se informa el inicio de esta adicción en edades adultas.

Con respecto al consumo de bebidas alcohólicas, se estima que su abuso representa $9 \%$ del costo total de la enfermedad en México, y se acompaña de padecimientos como cirrosis hepática, lesiones por accidentes de vehículos a motor, dependencia y homicidios. Además, se dispone de suficiente evidencia en cuanto a que los efectos del alcohol en jóvenes los hace más propensos que los adultos a observar comportamientos de alto riesgo, a la depresión e intento de suicidio, y a asumir conductas violentas. ${ }^{2}$ Aunado a estos efectos negativos en la salud, la información disponible comprueba de manera sobrada los enormes costos directos e indirectos por enfermedad o padecimientos relacionados con el hábito de fumar o consumir alcohol. $^{3}$

Muy diversas disciplinas estudian los factores que influyen en la demanda de tabaco y alcohol, o los factores que inciden en las decisiones de su consumo. Desde la perspectiva económica, se subrayan los efectos de variables económicas como los precios o regulaciones de mercado gubernamentales, no sólo porque es posible modelar la demanda utilizando las herramientas de la teoría económica, sino por su incidencia en la formulación de políticas públicas, en el supuesto de que el gobierno puede controlar estas variables con facilidad mediante diversas intervenciones.

No obstante, el fenómeno de las adiciones va más allá de una decisión "racional" de los individuos. Existe abundante información ${ }^{4-7}$ acerca de que la influencia de sus pares en los jóvenes ejerce un mayor efecto en el consumo de tabaco y alcohol que la interacción con adultos. No menos importante son las influencias de tipo familiar, escolar, comunitaria o de medios masivos de comunicación, que potencian los efectos de otros factores como los precios y la accesibilidad a los productos. Esta susceptibilidad social parece incrementarse en la adolescencia y declinar después de los 20 años de edad. Están presentes además otros aspectos de tipo psicosociall ${ }^{4,8}$ como cierto grado de desequilibro emocional, estrés, inteligencia emocional, etc., que influyen en la decisión de los individuos de consumir estas sustancias. Por otra parte, es también de importancia considerar la transformación tecnológica en la industria del tabaco, que la llevó a generar un instrumento de dosificación de nicotina de gran efectividad para suscitar adicción, además de las fuertes campañas de promoción para fomentar el consumo de 
tabaco entre jóvenes y mujeres. Diversos estudios han abordado la interacción entre las decisiones de fumar e ingerir alcohol. ${ }^{9-13}$ En una revisión sobre la literatura epidemiológica al respecto, ${ }^{14}$ se concluyó que la interacción en el consumo de tabaco y alcohol se debe a un amplio rango de factores biológicos, psicológicos y sociales, incluso se señala que los riesgos estimados a la salud por los efectos combinados del consumo de ambas sustancias es $50 \%$ mayor que la suma de los riesgos independientes.

En línea con la hipótesis de que las decisiones de los individuos por consumir ambas sustancias se influencian de manera recíproca a través de una serie de características observables y no observables, este análisis pretende identificar aquellos factores contextuales que determinan la decisión de su consumo.

\section{Material y métodos}

Para este análisis se analizó información de la Encuesta de Evaluación de los Hogares Urbanos 2002 (Encelurb 2002), * que constituye la línea basal de una encuesta de seguimiento para la evaluación de impacto de un programa social (Oportunidades) dirigido a familias que viven en pobreza extrema y que habitan en áreas urbanas de 50000 a un millón de habitantes. Se trató de un estudio cuasi experimental en el que se seleccionaron de manera probabilística ${ }^{\ddagger} 49$ manzanas (bloques de hogares) en las zonas donde el programa iniciaría operaciones en 2002. En las zonas donde el programa empezaría a operar en 2004 se seleccionaron 388 manzanas bajo un criterio de pareamiento (nearest neighbor matching method) que buscó maximizar la comparabilidad entre las manzanas de ambas zonas. La selección de los hogares se hizo en dos fases: en la primera, se seleccionaron todos los hogares de las manzanas escogidas con el fin de identificar su condición de elegibilidad e incorporación al programa (hogares elegibles, casi elegibles y no elegibles); $\$$ en la segunda fase, y con la información obtenida de la primera, se seleccionó la

\footnotetext{
* Personal con capacitación previa aplicó en los hogares la Encelurb, después de obtener el consentimiento informado de participación. Las comisiones de ética, bioseguridad e investigación del Instituto Nacional de Salud Pública aprobaron el estudio.

‡ La probabilidad de selección fue proporcional al número de hogares pobres residentes en ellas.

$\S$ Los hogares casi elegibles son aquellos que están inmediatamente por abajo del punto mínimo de corte que define a los hogares elegibles. El punto de corte fue determinado por los implementadores del programa y se hizo a partir de un análisis discriminante con información socioeconómica de los miembros del hogar y su vivienda.
}

muestra final de hogares para el estudio. Debido a este procedimiento de selección, la información captada en la Encelurb 2002 proviene de manera predominante de hogares elegibles, que son hogares en extrema pobreza y, en menor medida, de hogares menos pobres.

La Encelurb 2002 es una encuesta multitemática que captó información sobre las características sociodemográficas y económicas de 17207 hogares y de las personas que los componen, así como información sobre el consumo de tabaco y bebidas alcohólicas en 19624 jóvenes y adolescentes de 10 a 21 años de edad. Para efectos de este análisis se consideró sólo la información de los jóvenes de 12 a 21 años (15 181) debido a la muy baja prevalencia en el consumo de estas sustancias en el grupo de 10 y 11 años. Además de estos datos, se usó el índice de marginación urbana 2000 publicado por el Consejo Nacional de Población (CO$\mathrm{NAPO}$ ) con el fin de controlar la heterogeneidad observada por localidad.

Con el propósito de entender el patrón del consumo de tabaco y de bebidas alcohólicas de los jóvenes en estudio, se analizó de manera descriptiva la información referente al consumo de estas sustancias tal y como se captó en la encuesta. En la definición de prevalencia en el hábito de fumar se consideró como fumador aquel joven que aceptó haber fumado al menos un cigarrillo en los últimos 30 días (un mes) previos a la encuesta, en concordancia con la definición adoptada por los Centros para el Control y Prevención de Enfermedades de Estados Unidos (CDC, por sus siglas en inglés) en la Global Youth Tobacco Survey (GYTS) en 2003.* En el caso de la prevalencia en el consumo de bebidas alcohólicas se consideró como bebedor a aquel joven que reconoció haberlas ingerido aunque fuera en ocasiones; no resultó posible, entonces, establecer una definición más precisa de "bebedor regular o asiduo" debido a que no se contó con información más detallada sobre el consumo de bebidas alcohólicas.

Para explorar de manera descriptiva la interacción social de pares al interior del hogar se constituyeron dos grupos, uno formado por adolescentes de 12 a 15 años, y otro por jóvenes de 16 a 21 años de edad. ${ }^{\ddagger}$

\footnotetext{
* La GYTS es una encuesta internacional para la vigilancia del consumo de tabaco entre jóvenes de 12 a 15 años con el fin de guiar el desarrollo, implementación y evaluación de programas de prevención y control del tabaquismo.

‡ En 76\% de los casos se identificó una relación de hermanos y en casi 13\% no se identificó una relación de parentesco; por tanto, para este análisis, se consideraron a todos los jóvenes del hogar sin importar la relación de parentesco, después de asumir que la interacción entre ellos es igual.
} 
La intención de hacerlo fue comparar la prevalencia de fumadores de 12 a 15 años de edad en el caso de cohabitar con otros jóvenes mayores (16 a 21 años) también fumadores con los que cohabitaban con jóvenes mayores que manifestaron no fumar. El mismo ejercicio se replicó para el caso del consumo de bebidas alcohólicas.

Una vez que se definieron las prevalencias de fumar y del consumo de bebidas alcohólicas, se planteó el siguiente sistema de ecuaciones de acuerdo con el modelo que Maddala ${ }^{15}$ expuso:

$\mathrm{y}_{1}^{*}=\gamma_{1}^{\prime} \mathrm{x}_{1}+\mu_{1}$

$\mathrm{y}_{2}^{*}=\beta_{2} \mathrm{y}_{1}+\gamma_{2}^{\prime} \mathrm{x}_{2}+\mu_{2}$

donde $\mathrm{y}_{1}$ es la propensión a consumir bebidas alcohólicas y $\mathrm{y}_{2}$ es la propensión a fumar; ambas son variables latentes que toman los siguientes valores:

$\mathrm{y}_{1}=\quad 1$ si $\mathrm{y}_{1}>0$

$\mathrm{y}_{1}=0$ de otra manera

$\mathrm{y}_{2}=1$ si $\mathrm{y}_{2}>0$

$\mathrm{y}_{2}=0$ de otra manera

$\mathrm{X}_{1}$ y $\mathrm{X}_{2}$ representan los factores exógenos observables en el individuo, el hogar y la comunidad que afectan la decisión de consumir ambas sustancias

Dado que por hipótesis se supuso que $\mu_{1}$ y $\mu_{2}$ no son independientes, es decir, que ambas decisiones se afectan por características comunes no observables, se estimó el siguiente sistema de ecuaciones mediante un modelo probit bivariado:

$\mathrm{y}_{1}^{*}=\gamma_{1}{ }^{\prime} \mathrm{x}_{1}+\mu_{1}$

$\mathrm{y}_{2}{ }^{*}=\gamma_{2}{ }^{\prime} x_{2}+\mu_{2}$

con $\operatorname{Cov}\left(\mu_{1}, \mu_{1}\right) \neq 0$

Para probar la hipótesis de la interacción social al interior del hogar, se estimó el modelo con el uso de la submuestra de adolescentes de 12 a 15 años de edad, controlado por la presencia en el hogar de jóvenes de 16 a 21 años identificados como fumadores o consumidores de bebidas alcohólicas.*

En todas las estimaciones se incluyeron efectos fijos por entidad federativa con el fin de captar diferen-

\footnotetext{
* El 85\% de los jóvenes fumadores de 16 a 21 años de edad tuvo un mayor tiempo de exposición al tabaquismo que los adolescentes de 12 a 15 años, por lo que todos fueron considerados en el análisis, además de no poderse controlar por el tiempo de exposición al consumo de bebidas alcohólicas.
}

cias no medibles en ese nivel de gobierno, como podrían ser las políticas estatales de salud en el tema de las adicciones, o diferencias en las legislaciones estatales sobre uso y venta de tabaco y alcohol. De manera adicional, se incluyeron variables individuales como sexo, edad y estado marital, así como variables relacionadas con las características socioeconómicas del hogar que podrían afectar la propensión al consumo de ambas sustancias. Se incluyeron algunas variables demográficas del hogar como proxis del grado de socialización al interior del mismo, así como referentes al nivel socioeconómico. La inclusión de la escolaridad del jefe del hogar y su condición de indigenismo se eligió con el fin de captar la posible influencia de los padres en los hijos a través de un mayor conocimiento de los daños de fumar o beber alcohol en exceso. Se incluyó la condición de si los jóvenes vivían en el hogar con sus padres asumiendo que la presencia de ambos padres en el hogar ejerce un efecto de mayor control sobre el comportamiento de los hijos.

\section{Resultados}

La exploración descriptiva de la muestra (cuadro I) reveló que poco más de $85 \%$ de jóvenes manifestó contar con estudios de primaria o secundaria. Llama la atención que $11.32 \%$ no reconociera grado educativo alguno. Es de resaltar que cerca de $40 \%$ de los jóvenes no vive con sus dos padres, y que $11.7 \%$ no vive con ninguno de ellos. En cuanto al nivel socioeconómico del hogar donde habitan, la mayoría (64.09\%) vive en hogares en extrema pobreza, lo que se refleja también en el grado de marginalidad de la localidad donde residen, con cerca de $70 \%$ de los hogares establecidos en localidades de muy alta y alta marginación.

El cuadro II muestra que entre los jóvenes de 12 a 21 años de edad los hombres parecen estar más dispuestos a fumar que las mujeres; $22.5 \%$ de ellos mencionó haber fumado alguna vez aunque no se hubieran terminado el cigarrillo, lo que contrasta con el $8.9 \%$ de las mujeres que mencionaron haberlo hecho. De aquellos que reconocieron haber fumado el mes anterior a la entrevista, los hombres no sólo fumaron más días en promedio (12.41 días) que las mujeres (8.5 días), sino que el promedio de cigarrillos que fumaron por mes fue bastante mayor (18.3 contra 12.6 de las mujeres), y ambas diferencias resultaron estadísticamente significativas.

Es de destacar que en aquellos jóvenes que manifestaron haber fumado aunque fuera en ocasiones, los hombres y mujeres probaron su primer cigarrillo casi a la misma edad (15.18 años para los hombres, 15.05 para las mujeres), y la edad promedio de inicio del 
Estadísticas descriptivas de LOS JÓVENES

DE I 2 A 2 I AÑOS DE EDAD INCLUIDOS eN LA ENCELURB 2002. MÉxico 2002

Genera

$15|8|$

Por individuo

Promedio de edad en años

$15.85(2.805)^{*}$

Proporción de jóvenes solteros

84.52

Proporción de jóvenes de 12 a 15 años

Proporción de jóvenes de 16 a 21 años

49.32

50.68

Escolaridad del joven

$\%$ sin instrucción

11.32

$\%$ con primaria

45.48

$\%$ con secundaria

40.05

$\%$ con preparatoria o más

3.15

En el hogar

Proporción de jóvenes que viven con ambos padres 59.6

Proporción de jóvenes que viven con un padre

(padre o madre)

28.70

Proporción de jóvenes que no viven con sus padres

Promedio de personas en el hogar

1.69

Proporción con jefe de hogar hombre

$2.92(2.291)^{*}$

Jefe de hogar que habla lengua indígena

77.09

Proporción que reside en hogar pobre

12.26

Proporción que reside en hogar casi pobre

64.09

Proporción que reside en hogar no pobre

19.83

Proporción que reside en hogares con algún migrante

en los 12 meses previos a la encuesta

8.39

En la comunidad

Proporción que reside en localidades con grado

de marginación muy alta

35.04

Proporción que reside en localidades con grado

de marginación alta

34.52

Proporción que reside en localidades con grado

de marginación media o baja

30.43

* Desviación estándar

fumar asiduo también fue similar en ambos grupos (15.8 años para hombres, 15.7 para mujeres).

La prevalencia en el consumo de tabaco en hombres se incrementa con celeridad conforme aumenta la edad, de $1.08 \%$ en el grupo de adolescentes de 12 años a $24.25 \%$ en jóvenes de 17 años, hasta alcanzar una prevalencia de $36.3 \%$ en jóvenes de 21 años de edad (figu- ra 1). En el caso de las mujeres, el aumento de la prevalencia por edad creció en forma gradual y se mantuvo en torno a $5 \%$ para cada grupo de edad a partir de los 16 años.

En relación con el consumo de bebidas alcohólicas, el comportamiento de los hombres hacia su consumo fue muy similar al exhibido para el caso del consumo de tabaco. La prevalencia se incrementa con rapidez conforme aumenta la edad, de $2.2 \%$ en el grupo de adolescentes de 12 años a $43.6 \%$ en el grupo de jóvenes de 21 años de edad (figura 2). Si bien en el caso de las mujeres la prevalencia se mantuvo por debajo de $10 \%$ para cada grupo de edad, son más proclives a ingerir bebidas alcohólicas, aunque sea en ocasiones, que a fumar.

$\mathrm{Si}$ se toma en cuenta la clasificación de elegibilidad al programa Oportunidades como un buen referente del grado de pobreza de los hogares, se encontró que los jóvenes residentes de hogares más pobres o en pobreza extrema fueron menos propensos a fumar y a consumir bebidas alcohólicas que los jóvenes residentes de hogares menos pobres (cuadro III). Una vez que se es fumador, el promedio de cigarrillos diarios y el promedio de días que reportaron fumar al mes fue estadísticamente igual para los tres grupos, esto es, una vez iniciado el hábito de fumar, la intensidad en su consumo es la misma sin importar la condición económica de la familia.

Como se mencionó antes, la evidencia ha documentado ampliamente la importancia que encierra la interacción social de los jóvenes con su entorno en el consumo de diversas sustancias adictivas. De manera descriptiva, el cuadro IV muestra que la prevalencia en el consumo de tabaco en jóvenes de 12 a 15 años de edad que cohabitaban con jóvenes de 16 a 21 años que fumaban resultó mucho mayor $8.7 \%$ ) que la de aquellos que compartían el hogar con jóvenes de 16 a 21 años no fumadores (1.6\%).* De igual forma, la prevalencia en el consumo de bebidas alcohólicas en adolescentes de 12 a 15 años fue mayor si habitaban

\footnotetext{
* Para cada hogar se identificó primero la presencia de jóvenes de 12 a 15 años; luego se indagó la presencia o no de jóvenes de 16 a 21 años y, de haberlos, se averiguó quiénes fumaban o no; un ejercicio similar se hizo para el caso del consumo de bebidas alcohólicas. Para el consumo de tabaco, la comparación se llevó a cabo considerando sólo aquellos casos donde los jóvenes mayores (16 a 21 años) fumadores tenían igual o mayor tiempo de haberse iniciado en el fumar que los adolescentes de 12 a 15 años. Esta condición se dio en 45 de 53 casos, es decir, 85\% de los adolescentes empezaron a fumar después ( 40 casos) o al mismo tiempo (5 casos) que los jóvenes mayores fumadores en el hogar. En el caso del consumo de bebidas alcohólicas no fue posible establecer esta separación debido a la falta de información.
} 
Cuadro II

Descripción del consumo de tabaco y Alcohol Por SEXo. México 2002

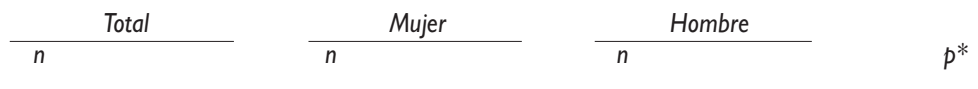

Consumo de tabaco

\begin{tabular}{|c|c|c|c|c|c|c|c|}
\hline Proporción que alguna vez ha fumado & 2370 & $15.7 \mid$ & 675 & 8.94 & 1695 & 22.51 & 0.000 \\
\hline Edad promedio de prueba del primer cigarrillo & 2258 & $15.08(2.38)^{\ddagger}$ & 628 & $15.18(2.39)^{\ddagger}$ & 1630 & $15.05(2.37)^{\ddagger}$ & 0.318 \\
\hline Edad promedio de inicio del fumar asiduo & 670 & $15.80(2.24)^{\ddagger}$ & 112 & I5.7I $(2.03)^{\ddagger}$ & 558 & $15.82(2.28)^{\ddagger}$ & 0.616 \\
\hline Promedio de días que fuma & 1240 & $\mathrm{II} .83(\mathrm{II} .24)^{\ddagger}$ & 186 & $8.52(10.44)^{\ddagger}$ & 1054 & $12.4 \mathrm{I}(\mathrm{II} .28)^{\ddagger}$ & 0.000 \\
\hline Promedio de cigarrillos que fuma & $\mathrm{I} 122$ & $17.40(21.69)^{\ddagger}$ & 176 & $12.59(19.84)^{\ddagger}$ & 946 & $18.29(21.92)^{\ddagger}$ & 0.001 \\
\hline Prevalencia en consumo de tabaco & 1256 & 8.60 & 189 & 2.58 & 1067 & 14.69 & 0.000 \\
\hline
\end{tabular}

Consumo de bebidas alcohólicas

\begin{tabular}{llllll|l} 
Prevalencia en consumo de bebidas alcohólicas & I 662 & II.29 & 467 & 6.35 & I & I
\end{tabular}

* Nivel de significancia estadística en la diferencia por sexo. Se ajustaron los errores estándar por cluster a nivel de manzana

‡ Desviación estándar

Fuente: Encelurb 2002

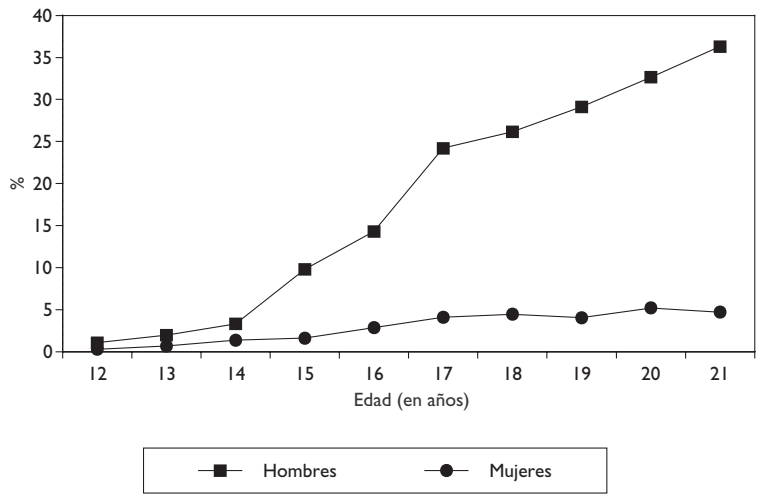

Figura I. Prevalencia en el consumo de tabaco por EDAD Y SEXO. MÉXICO 2002

con jóvenes de 16 a 21 años que manifestaron consumirlas que en aquellos que compartían el hogar con jóvenes de 16 a 21 años que eran abstemios (11.3\% contra $2.6 \%)$.

\section{Determinantes socioeconómicos de la prevalencia de fumar y consumo de bebidas alcohólicas}

Los resultados de regresión obtenidos por el modelo probit bivariado se muestran en el cuadro V. Para la



Figura 2. Prevalencia en el consumo de bebidas alCOHÓLICAS POR EDAD Y SEXO. MÉXICO 2002

muestra total y la submuestra de análisis, los coeficientes de correlación entre los términos de error del conjunto de ecuaciones (1) y (2) fueron positivos y estadísticamente significativos, en tanto que la prueba de Wald rechaza la hipótesis de que la correlación sea cero en los dos casos, lo que corrobora la hipótesis previamente planteada de que las decisiones de los jóvenes de fumar y/o consumir bebidas alcohólicas guardan una relación estrecha mediante una serie de factores observables y no observables, aun cuando no se establezca una relación causal entre 
Cuadro III

Descripción del CONSUMO de tABACO y ALCOHOL POR CONDICIÓn DE POBREZA. MéXico 2002

\begin{tabular}{|c|c|c|c|c|c|c|c|c|}
\hline & \multicolumn{2}{|r|}{ Pobre } & \multicolumn{2}{|r|}{ Casi pobre } & \multirow[b]{2}{*}{$p^{*}$} & \multicolumn{2}{|r|}{ No pobre } & \multirow[b]{2}{*}{$p^{*}$} \\
\hline & $n$ & & $n$ & & & $n$ & & \\
\hline \multicolumn{9}{|l|}{ Consumo de tabaco } \\
\hline Proporción que alguna vez ha fumado & 1340 & 13.85 & 524 & 17.53 & 0.000 & 506 & 20.91 & 0.000 \\
\hline Edad promedio de prueba del primer cigarrillo & | 27| & $15.00(2.38)^{\ddagger}$ & 500 & $15.04(2.39)^{\ddagger}$ & 0.782 & 487 & $15.36(2.34)^{\ddagger}$ & 0.014 \\
\hline Edad promedio de inicio del fumar asiduo & 393 & $15.65(2.27)^{\ddagger}$ & 147 & $15.75(2.35)^{\ddagger}$ & 0.623 & 130 & $16.3(1.90)^{\ddagger}$ & 0.002 \\
\hline Promedio de días que fuma & 708 & $12.09(\mid 1.27)^{\ddagger}$ & 262 & $11.39(10.86)^{\ddagger}$ & 0.406 & 270 & II.58 (II.53) & 0.586 \\
\hline Promedio de cigarrillos que fuma & 640 & $18.04(22.00)^{\ddagger}$ & 247 & $15.98(19.54)^{\ddagger}$ & 0.225 & 235 & $17.17(22.97)^{\ddagger}$ & 0.653 \\
\hline Prevalencia en consumo de tabaco & 717 & 7.62 & 265 & 9.21 & 0.017 & 274 & 11.80 & 0.000 \\
\hline \multicolumn{9}{|l|}{ Consumo de bebidas alcohólicas } \\
\hline Prevalencia en consumo de bebidas alcohólicas & 925 & 9.84 & 379 & 12.90 & 0.000 & 358 & $15.0 \mid$ & 0.000 \\
\hline \multicolumn{9}{|c|}{$\begin{array}{l}\text { * Nivel de significancia estadística en la diferencia por grupos (grupo de hogares pobres como grupo de referencia). Se ajustaron los errores estándar por } \\
\text { cluster a nivel de manzana } \\
\text { ‡ Desviación estándar entre paréntesis }\end{array}$} \\
\hline
\end{tabular}

\section{Cuadro IV \\ Prevalencias cruzadas de fumar Y BEBER ALCOHOL ENTRE GRUPOS DE EDAD. MÉXICO}

\begin{tabular}{|c|c|c|c|c|}
\hline & & $\begin{array}{l}\text { habita } \\
16 \text { a }\end{array}$ & $\begin{array}{l}\text { n jóve } \\
\text { años }\end{array}$ & \\
\hline & & & & uman \\
\hline & $n$ & $\%$ & $n$ & $\%$ \\
\hline $\begin{array}{l}\text { Prevalencia en consumo de tabaco } \\
\text { en jóvenes de } 12 \text { a } 15 \text { años }\end{array}$ & 45 & 8.7 & 45 & 1.63 \\
\hline & & & & eben \\
\hline & $n$ & $\%$ & $n$ & $\%$ \\
\hline $\begin{array}{l}\text { Prevalencia en consumo de alcohol } \\
\text { en jóvenes de } 12 \text { a } 15 \text { años }\end{array}$ & 82 & II.33 & 68 & 2.65 \\
\hline ente: Encelurb 2002 & & & & \\
\hline
\end{tabular}

ellas. Esta interdependencia se observa mejor en el hecho de que la mayoría de las características individuales y del hogar detentaran signo y significancia similares en su asociación con la propensión al consumo de ambas sustancias.

Como en otros estudios llevados a cabo en jóvenes, ${ }^{10,16}$ en ambas poblaciones se observa que los hombres tienen una mayor probabilidad de ser fumadores o de consumir bebidas alcohólicas que las mujeres; más aún, este efecto es más acentuado conforme aumenta la edad, tal y como se muestra en las figuras 1 y 2 . Por otro lado, los hombres que están casados o unidos tie- nen una mayor probabilidad de consumir ambas sustancias.

En cuanto a las características del hogar y su relación con la propensión a fumar o ingerir bebidas alcohólicas, se encontró que si los jóvenes no viven en el hogar con sus padres la probabilidad de consumir ambas sustancias es mayor que si vivieran con ambos padres. Además, cuantas más horas diarias de televisión, mayor probabilidad de los jóvenes del hogar de fumar o beber alcohol. Esta relación sólo fue significativa si el hogar estuvo más de cinco horas diarias frente al televisor respecto a los hogares que manifestaron no ver televisión. Una probabilidad mayor de fumar y/o ingerir alcohol también se asoció con la presencia en el hogar de tres o más personas laboralmente activas, en contraste con hogares con ningún trabajador activo. Por otro lado, una menor probabilidad en el consumo de ambas sustancias acompañó a la condición de habla indígena del jefe o jefa del hogar. Una asociación similar se encontró en el caso de los jóvenes que habitaban en hogares residentes en localidades de alta y muy alta marginalidad con respecto a aquellos que vivían en localidades de media o baja marginación.

Por otro lado, no se encontró evidencia de una asociación significativa de la composición demográfica del hogar ni de algunas características propias del jefe del hogar, como su educación, sexo o edad, en la propensión al consumo de tabaco o alcohol.

Una de las hipótesis a probar en este análisis fue la influencia de otros jóvenes de mayor edad en el ho- 


\section{Cuadro V \\ Resultados del modelo de Regresión Probit bivariado. México 2002}

\begin{tabular}{|c|c|c|c|c|}
\hline \multirow[b]{2}{*}{ Variables } & \multicolumn{2}{|c|}{ Jóvenes de 12 a 21 años de edad } & \multicolumn{2}{|c|}{$\begin{array}{c}\text { Jóvenes de } 12 \text { a } 15 \text { años de edad } \\
2\end{array}$} \\
\hline & $\begin{array}{l}\text { Propensión } \\
\text { a fumar }\end{array}$ & $\begin{array}{c}\text { Propensión } \\
\text { a consumir alcohol }\end{array}$ & $\begin{array}{l}\text { Propensión } \\
\text { a fumar }\end{array}$ & $\begin{array}{c}\text { Propensión } \\
\text { a consumir alcohol }\end{array}$ \\
\hline \multirow[t]{2}{*}{ sexo $(I=$ hombre, $0=$ mujer $)$} & 0.496 & 0.044 & 0.446 & 0.043 \\
\hline & {$[4.86]^{*}$} & {$[0.63]$} & {$[4.19]^{*}$} & [0.62] \\
\hline \multirow[t]{2}{*}{ edad I ( $I=15$ y 16 años, $0=12$ a $\mid 4$ años $)$} & 0.428 & 0.277 & 0.262 & 0.335 \\
\hline & {$[3.87]^{*}$} & {$[3.74]^{*}$} & {$[1.95]^{\ddagger}$} & {$[3.68]^{*}$} \\
\hline \multirow[t]{2}{*}{ edad 2 ( $I=17$ y 18 años $)$} & 0.67 & 0.495 & & \\
\hline & {$[6.54]^{*}$} & {$[6.32]^{*}$} & & \\
\hline \multirow[t]{2}{*}{ edad 3 ( $I=19$ a $2 I$ años) } & 0.632 & 0.56 & & \\
\hline & {$[5.19]^{*}$} & {$[7.34]^{*}$} & & \\
\hline \multirow{2}{*}{ sexo por edad I } & 0.367 & 0.427 & 0.43 & 0.223 \\
\hline & {$[2.92]^{*}$} & {$[4.13]^{*}$} & {$[3.10]^{*}$} & {$[1.99]^{\S}$} \\
\hline \multirow[t]{2}{*}{ sexo por edad 2} & 0.645 & 0.655 & & \\
\hline & {$[5.66]^{*}$} & {$[6.17]^{*}$} & & \\
\hline \multirow[t]{2}{*}{ sexo $\ddagger$ por edad 3} & 0.754 & 0.829 & & \\
\hline & {$[5 . \mathrm{II}]^{*}$} & {$[7.60]^{*}$} & & \\
\hline \multirow[t]{2}{*}{ estado marital $(I=$ otro, $0=$ soltero $)$} & 0.153 & 0.092 & & \\
\hline & [1.34] & {$[1.15]$} & & \\
\hline \multirow[t]{2}{*}{ sexo marital } & 0.244 & 0.275 & & \\
\hline & {$[2.47]^{\S}$} & {$[3.09]^{*}$} & & \\
\hline \multirow[t]{2}{*}{ número de personas en $\mathrm{HH}$} & -0.04 & -0.027 & 0.014 & 0.00 \\
\hline & {$[2.15]^{\S}$} & {$[1.69]^{\ddagger}$} & {$[0.45]$} & {$[0.01]$} \\
\hline \multirow[t]{2}{*}{ jefe $\mathrm{HH}$ habla lengua indígena } & -0.118 & -0.168 & 0.082 & -0.253 \\
\hline & {$[1.91]^{\ddagger}$} & {$[2.57]^{\S}$} & {$[0.82]$} & {$[2.07]^{\S}$} \\
\hline \multirow{2}{*}{ vive con padres ( $I=$ con un padre, $0=$ con ambos $)$} & 0.27 & 0.208 & 0.234 & 0.19 \\
\hline & {$[4.44]^{*}$} & {$[3.75]^{*}$} & {$[2.39]^{\S}$} & {$[1.90]^{\ddagger}$} \\
\hline \multirow[t]{2}{*}{ vive con padres (I = no vive con sus padres) } & 0.199 & 0.268 & 0.53 & 0.225 \\
\hline & {$[2.05]^{\S}$} & {$[3.95]^{*}$} & {$[4.31]^{*}$} & {$[1.44]$} \\
\hline \multirow[t]{2}{*}{ personas en el $\mathrm{HH}$ con trabajo remun. ( $(=$ una, $0=$ ninguna $)$} & 0.004 & 0.095 & 0.172 & 0.14 \\
\hline & {$[0.04]$} & [0.78] & {$[1.02]$} & [0.78] \\
\hline \multirow[t]{2}{*}{ personas en el $\mathrm{HH}$ con trabajo remun. (I= dos trabajadores) } & 0.139 & 0.172 & 0.128 & 0.1 \\
\hline & {$[\mathrm{I} .4 \mathrm{I}]$} & {$[1.38]$} & {$[0.69]$} & [0.55] \\
\hline \multirow[t]{2}{*}{ personas en el $\mathrm{HH}$ con trabajo remun. $(\mathrm{I}=$ tres a más) } & 0.237 & 0.227 & 0.231 & 0.173 \\
\hline & {$[2.53]^{\S}$} & {$[1.83]^{\ddagger}$} & {$[1.19]$} & {$[0.85]$} \\
\hline \multirow[t]{2}{*}{ horas diarias de TV por $\mathrm{HH}(\mathrm{I}=\mathrm{I} \circ 2,0=$ ninguna $)$} & -0.089 & -0.029 & -0.069 & -0.01 \\
\hline & {$[1.31]$} & {$[0.57]$} & {$[0.47]$} & {$[0.10]$} \\
\hline \multirow[t]{2}{*}{ horas diarias de TV por $\mathrm{HH}(\mathrm{I}=3$ a 5$)$} & 0.016 & 0.063 & -0.013 & 0.068 \\
\hline & {$[0.24]$} & {$[1.29]$} & {$[0.10]$} & {$[0.73]$} \\
\hline \multirow[t]{3}{*}{ horas diarias de TV por HH (I= más de 5) } & 0.31 & 0.269 & 0.294 & 0.173 \\
\hline & {$[3.65]^{*}$} & {$[3.46]^{*}$} & {$[1.97]^{\S}$} & {$[1.40]$} \\
\hline & {$[1.38]$} & {$[0.31]$} & {$[1.80]^{\ddagger}$} & {$[1.58]$} \\
\hline \multirow[t]{2}{*}{ marginación localidad ( $\mathrm{I}=$ alta, $0=$ media $\circ$ baja $)$} & -0.177 & -0.163 & -0.222 & -0.258 \\
\hline & {$[2.73]^{*}$} & {$[2.43]^{\S}$} & {$[2.37]^{\S}$} & {$[3.95]^{*}$} \\
\hline marginación localidad ( $I=$ muy alta) & -0.23 & -0.044 & -0.097 & 0.004 \\
\hline & {$[3.10]^{*}$} & {$[0.65]$} & {$[1.00]$} & {$[0.04]$} \\
\hline otros fumadores $(I=\sin$ mayores, $0=$ con mayores no fumadores $)$ & & & 0.128 & 0.193 \\
\hline & & & {$[1.15]$} & {$[2.52]^{\S}$} \\
\hline
\end{tabular}


Continuación

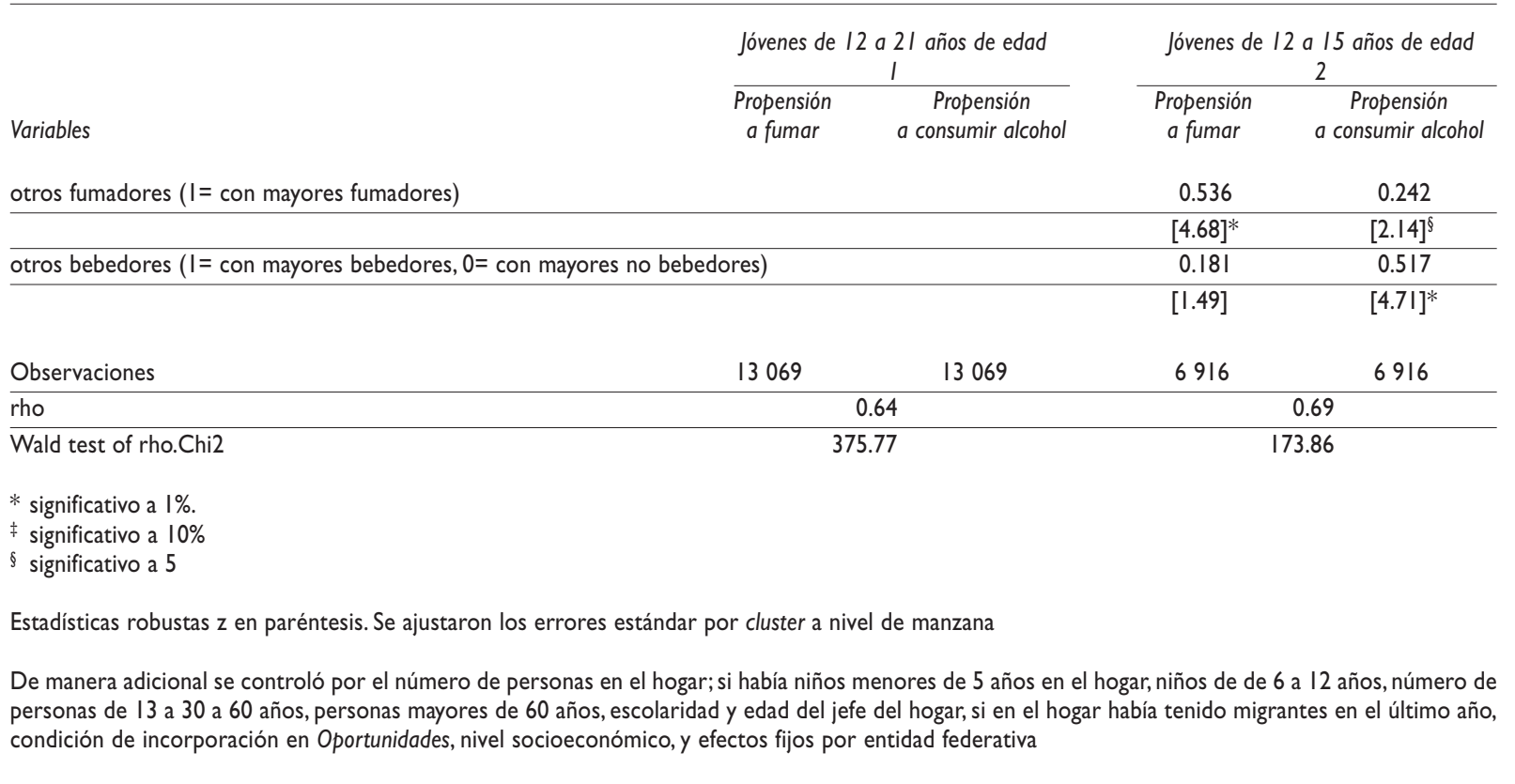

gar, sobre la probabilidad de fumar o beber alcohol en adolescentes y/o jóvenes menores. Los resultados del análisis de regresión con la submuestra de adolescentes de 12 a 15 años muestran que la presencia de jóvenes fumadores de entre 16 y 21 años de edad, guardó relación con una mayor propensión a fumar en los adolescentes de 12 y 15 años que en aquellos casos donde, a pesar de verificarse la presencia de jóvenes mayores en el hogar, éstos no fumaban (cuadro V, columna 2). En el mismo sentido, la presencia de jóvenes de 16 a 21 años que reconocieron ingerir alcohol mostró una asociación significativa con una mayor probabilidad de que los adolescentes de 12 a 15 años también lo consumieran. Con estos resultados, la razón de prevalencias estimadas* en el consumo de tabaco en aquellos de 12 a 15 años fue 2.76 veces mayor si éstos cohabitan con jóvenes (16 a 21 años) fumadores que si cohabitan con jóvenes de 16 a 21 años no fumadores, y la correspondiente al consumo de alcohol para el mismo grupo de edad fue 2.54 veces mayor si en el hogar hay otros jóvenes de 16 a 21 años que ingieren alcohol que si cohabitan con jóvenes de la misma edad que no lo

\footnotetext{
* La razón de prevalencias se estimó sólo para aquellos casos donde habitaban en el hogar jóvenes de 16 a 21 años de edad.
}

ingieren. El método de estimación permitió calcular la probabilidad conjunta de consumir ambas sustancias, en este caso, la probabilidad de que los adolescentes de 12 a 15 años fumen e ingieran bebidas alcohólicas en presencia de otros jóvenes de mayor edad en el hogar consumidores de ambas sustancias fue 5.62 veces mayor que si esos otros jóvenes de mayor edad no las consumen.

La asociación de la propensión a fumar y/o consumir alcohol con otras características individuales y familiares para la submuestra de análisis fue, en los casos donde hubo significancia estadística, consistente en signo y magnitud con los hallazgos del análisis con la muestra completa (12 a 21 años de edad).

\section{Discusión}

Los resultados de este estudio ponen de manifiesto que, entre los jóvenes de zonas urbanas marginadas de México, las decisiones de consumir tabaco y alcohol no son independientes, y que la probabilidad en el consumo de ambas sustancias por jóvenes de 12 a 15 años es mayor si cohabitan con jóvenes mayores en edad que las consuman.

En general, los resultados exploratorios son consistentes con lo encontrado en México en otras encuestas como la Encuesta Nacional de Adicciones 2002 
(ENA 2002) y la Encuesta sobre Tabaquismo en Jóvenes 2003 (ETJ 2003). 17,18,* De acuerdo con la ENA 2002, que tiene representatividad nacional, se encontró que los hombres son más proclives a fumar que las mujeres, aun cuando en encuestas como la ETJ 2003 esta diferencia ya no muestra ser significativa. En relación con el consumo de bebidas alcohólicas, la prevalencia fue notoriamente mayor en hombres $(16.2 \%)$ que en mujeres $(6.35 \%)$; no obstante, estas prevalencias ${ }^{\ddagger}$ fueron menores a las encontradas en la ENA 2002, donde la prevalencia en hombres de 15 a 17 años fue de $35 \%$ y en mujeres, de $25 \%$. Para ambos sexos, el incremento de las prevalencias en el consumo de tabaco y bebidas alcohólicas por edad no es lineal, lo que resulta consistente con lo que revelan otros estudios internacionales. ${ }^{6,19}$ Es probable que conforme adquieren mayor edad los jóvenes se encuentran mejor orientados hacia el futuro y reconocen con más claridad los riesgos de fumar o consumir alcohol, como lo sugieren el enfoque sociológico de las conductas de riesgo o la economía conductual. ${ }^{4,20,21}$

Resulta alarmante observar el rápido incremento en las prevalencias del consumo de tabaco y alcohol en hombres menores de edad, aun cuando la venta de estas sustancias adictivas es ilegal antes de los 18 años, lo que pone en evidencia que en la práctica las regulaciones al respecto adolecen de implementación deficiente. Más aún, el tiempo transcurrido entre que se probó el cigarrillo y se empezó a fumar en forma asidua (casi a diario) fue menor de un año, lo que sugiere una formación de la adicción bastante rápida.

Los resultados de regresión son consistentes con lo encontrado en otros estudios, ${ }^{9-14}$ y confirman la hipótesis de que intervienen factores observables y no observables que determinan que las decisiones de los jóvenes de fumar o consumir bebidas alcohólicas guarden una relación estrecha. Este resultado es importante porque apunta a la necesidad de profundizar en el entendimiento de esta relación por las implicaciones que tiene en la formulación de políticas públicas tendientes a disminuir su consumo. En este sentido, por el lado de las variables de mercado, se hace indispen-

\footnotetext{
* La ETJ 2003 se llevó a cabo en jóvenes de secundaria de 10 ciudades importantes del país (Tijuana, Ciudad Juárez, Nuevo Laredo, Guadalajara, Cuernavaca, Puebla, Ciudad de México, Oaxaca, Tapachula y Chetumal).

$\ddagger$ Las comparaciones en este rubro se sugiere tomarlas con reserva, debido a que la referencia en la ENA es si los jóvenes tomaron una copa completa de bebida alcohólica en el año previo a la encuesta.
}

sable estudiar la manera como estas demandas se relacionan a través de los precios. Si se prueba que ambos bienes son sustitutos, entonces implicaría replantear el impuesto óptimo que tendría que aplicarse a cada uno de estos bienes sin que la medida repercuta en una mayor demanda del otro; por otro lado, si estos bienes son complementarios, las medidas restrictivas a la demanda de uno de ellos tendrían efectos indirectos en la demanda del otro similar a una sinergia y, en consecuencia, un efecto multiplicador en la reducción de la demanda de ambos. No menos importante resulta identificar el grado de simetría en los efectos de los precios cruzados ya que, como lo señalan en algunos estudios, ${ }^{9-13}$ la sensibilidad de la demanda de cada uno de estos bienes es diferente y ello repercute de manera importante en el diseño de la política impositiva. Dado que no está claro en la experiencia internacional qué tipo de relación guardan estas demandas, es necesario entender esta relación para diferentes poblaciones en México.

En el análisis de las interacciones sociales al interior del hogar se encontró que la probabilidad de que los jóvenes fumen y/o consuman alcohol fue significativamente mayor si en el hogar hay otros jóvenes (pares), en este caso mayores en edad, que también consumen estas sustancias adictivas. Este resultado subraya la importancia del papel familiar en las decisiones de los jóvenes de consumir estas sustancias adictivas como una forma de externalidad social de la información. En este sentido, se conoce ${ }^{7}$ que los jóvenes actualizan sus preferencias y aprenden sobre los riesgos y beneficios de consumir ciertos bienes a través de la conducta de otros jóvenes en la familia.

Entre las limitaciones de este estudio se reconoce la de no contar con información longitudinal, lo que impide analizar la dinámica en la demanda de estas sustancias y probar otras hipótesis más relacionadas con el grado de adicción alcanzado y su influencia en la demanda actual. Cabe destacar que los resultados que aquí se presentan no son generalizables a toda la población pobre de México, ya que los propósitos para los cuales se seleccionó la muestra resultaron en un sobremuestreo de los hogares más pobres residentes en áreas urbanas ( 50 mil a un millón de habitantes) del país. Ello hace necesario replicar el análisis con otras poblaciones o muestras con representatividad nacional antes de poder sacar conclusiones definitivas sobre ciertos efectos. Otra limitación importante fue carecer de más información sobre el consumo de bebidas alcohólicas que permitiera definir con mayor precisión la prevalencia de "bebedor habitual", sin incluir aquellos que pudieron haber bebido alcohol de manera experimental. 
Si bien los resultados de este análisis son modestos, su contribución es importante ya que permite identificar mejor aquellos factores individuales y familiares que inciden en la propensión de los jóvenes a fumar y/ o consumir bebidas alcohólicas, además de sumarse a la escasa literatura en México al respecto ${ }^{22}$ lo que se espera contribuya en el futuro a modelar mejor su demanda. Aunque aquí sólo se estudió la propensión al consumo de sustancias legales, existe la posibilidad de que ambas decisiones también guarden relación con otras drogas ilegales.

Por el lado de los factores sociodemográficos, la posibilidad de contar con valiosa información del hogar permitió a los autores probar, por un lado, la hipótesis de la influencia negativa que pueden ejercer los pares, en este caso otros jóvenes en el hogar que fuman o beben alcohol, en el comportamiento de los jóvenes de menor edad frente a estas sustancias y, por otro, los efectos positivos de residir en hogares con familia integrada, con padre y madre, sobre este tipo de comportamiento. Estos resultados tienen importantes implicaciones para el diseño e implementación de programas de control y prevención de adicciones, ya que revelan el importante papel que debe desempeñar la familia en las campañas de control y prevención.

\section{Referencias}

I. Secretaría de Salud. Programa de Acción:Adicciones Tabaquismo 2001. México, DF: SSA, 200I.

2. Medina-Mora $\mathrm{M}$ et al. Alcoholismo y abuso de bebidas alcohólicas. En: Observatorio mexicano en tabaco, alcohol y otras drogas. México, DF: Secretaría de Salud, 2002.

3. Research for International Tobacco Control 2003. At what cost?. The economic impact of tobacco use on national health systems societies and individuals: a summary of methods and findings. Monograph Series No. I. Otawa, Canada: RITC, 2003.
4. Gruber J. Risky behavior among youths: an economic analysis. NBER 2000, Working Paper 778I.

5. Case A, Katz L. The company you keep: the effects of family and neighborhood on disadvantaged youths. NBER 1991, Working Paper 3705. 6. Andrew C, Loheac Y."It wasn't me, it was them!" Social influence in risky behavior by adolescents. Royal Economic Society Annual Conference 2003; I-2I.

7. Jeffrey HE, Gonzalez LB.Asymmetric social interaction in economics: Cigarette smoking among young people in the United States, 1992-1999. NBER 2004, Working Paper 10409.

8. Organización Panamericana de la Salud. Por una juventud sin tabaco. Adquisición de habilidades para una vida saludable. Publicación Científica y Técnica 200I; 579.

9. Rajeev G, Mathew M. The interdependence of cigarette and liquor demand. South Econ J 1995;62:45I-459.

10. Decker S, Schwartz A. Cigarettes and alcohol: substitutes or complements? NBER 2000, Working Paper 7535.

II. Jones A. A systems approach to the demand for alcohol and tobacco. Bull Econ Res 1989;41:85-105.

12. Cameron L,Williams J. Cannabis, alcohol and cigarettes: substitutes or complements? Econ Rec 2001;77:19-34.

13. Manrique J, Jensen H. Consumption of tobacco and alcoholic beverages among Spanish consumers. Southwest Econ Rev 2004;3I: 4I-56.

14. Bien T et al. Smoking and drinking: a review of the literature. Int J Addic 1990;25: 1429-1454.

15. Maddala GS. Limited dependent and qualitative variables in Econometrics. Cambridge University Press: New York, 1983.

16. Chaloupka F,Warner K. The economics of smoking. En: Culyer AJ \& Newhouse JP, eds. The Handbook of Health Economics. vol. I. Ch. 29, 2000. 17. Secretaría de Salud. Encuesta Nacional de Adicciones 2002, Tabaco y alcohol y otras drogas. Resumen ejecutivo 2002. México, DF: SSA, 2002. 18.Valdés-Salgado $R$ et al. Encuesta sobre Tabaquismo en Jóvenes, México 2003. Cuernavaca, México: Instituto Nacional de Salud Pública, 2004. 19. Chaloupka F. Men, women, and addiction: the case of cigarette smoking. NBER 1990, Working Paper 3267.

20. Fehr E, Zych P. Do addicts behave rationally? Scand J Econ 2000;100:643-662.

21. Slovic P. What does it jeans to know a cumulative risk? Adolescents's perceptions of short term and long term consequences of smoking.J Behav Dec Making 2000;13:259-266.

22. Medina Mora $M$ et al. Del tabaco al uso de otras drogas: iel uso temprano de tabaco aumenta la probabilidad de usar otras drogas?. Salud Publica Mex 2002;44 Supl I:SI09-SII5. 